\title{
Families of Sections of Quadrics and Classical Geometries*
}

\section{A. G. Khovanskii}

UDC $514.13+519.675$

To the memory of my father Georgii Sergeevich Khovanskii

In the note we describe all Riemannian metrics on nondegenerate quadrics whose geodesics are plane curves. It is shown that any such metric is of constant curvature and hence locally defines one of the classical geometries on the quadric. The present note is the continuation of [5], where the problem of rectification of circles was solved. This practical problem was posed by G. S. Khovanskii in connection with his work on the transformation of nomograms of adjusted points into compass nomograms [1-4]).

1. Quadric points of a projective surface. A point $A$ on a germ of a real regular surface in a real projective space is said to be quadric if there is a quadric that anomalously closely approximates the surface at this point, i.e., the distance from a point $B$ on the surface to the quadric is an infinitesimal of order four with respect to the distance from $A$ to $B$.

We say that an affine coordinate system $x, y, z$ in an affine neighborhood of the projective space is adapted to a surface at a point $A$ if the origin coincides with this point and the plane $z=0$ is tangent to the surface at $A$. Suppose that a local equation of the surface in a neighborhood of $A$ in some adapted coordinate system has the form $z=f(x, y)=B_{2}(x, y)+K_{3}(x, y)+\ldots$, where $B_{2}$ and $K_{3}$ are the quadratic and the cubic terms of the Taylor series of $f$ at the origin, respectively.

Lemma 1. (1) The point $A$ is a quadric point of the surface if and only if the cubic polynomial $K_{3}$ is divisible by the second-degree polynomial $B_{2}$.

(2) The condition that the polynomial $K_{3}$ is divisible by the polynomial $B_{2}$ is projectively invariant, i.e., independent of the choice of an adapted coordinate system.

(3) The point $A$ is a quadric point of the surface if and only if there is an adapted coordinate system for which the polynomial $K_{3}$ is identically zero.

Proof. (1) Let $K_{3}=L_{1} B_{2}$, where $L_{1}$ is a homogeneous linear polynomial in $x$ and $y$. Then the surface is anomalously closely approximated by the quadric $z=B_{2}+L_{1} z$. Conversely, any quadric tangent to the plane $z=0$ at zero is given by an equation of the form $z=B_{2}+L_{1} z+c z^{2}$, where $B_{2}$ and $L_{1}$ are homogeneous polynomials in $x$ and $y$ of degrees one and two, respectively, and $c$ is a constant. Solving this equation up to the cubic terms inclusively, we obtain $z=B_{2}+L_{1} B_{2}+\ldots$. Assertion (2) readily follows from assertion (1). One of the implications in assertion (3) is also an immediate consequence of (1). To prove the converse implication, it suffices to choose an adapted coordinate system satisfying the following additional conditions: the plane at infinity coincides with the tangent plane of the approximating quadric at some point of the quadric other than $A$, and the $z$ axis passes through this point at infinity. In these coordinates, the approximating quadric is given by the equation $z=B_{2}(x, y)$, and the polynomial $K_{3}$ vanishes.

A nondegenerate quadric intersects a tangent plane to itself by a pair of lines, real or complex. We can readily verify the following assertion.

Lemma 2. A point $A$ of a germ of a strictly hyperbolic surface is quadric if and only if each of the two branches of the intersection of the surface with the tangent plane at $A$ has an inflection at this point. A point $A$ of a germ of a strictly elliptic surface is quadric if and only if each of the two complex branches of

*This research was partially supported by RFBR grant No. 99-01-00245 and by Canadian grant No. 156833-98.

Institute for System Studies of the Russian Academy of Sciences and the University of Toronto, Canada. Translated from Funktsional' nyi Analiz i Ego Prilozheniya, Vol. 34, No. 3, pp. 91-95, July-September, 2000. Original article submitted December 16, 1999. 
the intersection of the complexification of the 3-jet of the surface with the complex tangent plane at $A$ has an inflection at this point.

Remark. The definition of a quadric point can automatically be extended to complex surfaces in a complex projective space. The following problem is of interest in my opinion: How many quadric points does a generic surface of degree $n$ have in a complex projective space?

2. Rectifiable families of plane sections. In a neighborhood of the zero point on the plane, we consider a germ of a smooth function $F$ whose Taylor series expansion up to third-order terms is $F(x, y)=$ $\mu x-y+P_{2}(x, y)+P_{3}(x, y)+\ldots$, where $\mu$ is a number and $P_{2}$ and $P_{3}$ are homogeneous polynomials of degrees 2 and 3, respectively. The following Lemma 3 can be verified by straightforward computation.

Lemma 3. Up to terms of order three, the solution $y(x)$ of the equation $F(x, y(x))=0$ is given by the formula

$$
y(x)=\mu x+P_{2}(1, \mu) x^{2}+\left[\frac{\partial P_{2}}{\partial y}(1, \mu) P_{2}(1, \mu)+P_{3}(1, \mu)\right] x^{3}+\ldots
$$

Corollary 1. In the local coordinates $x, y$ on the surface, the intersection of the surface $z=f(x, y)=$ $B_{2}(x, y)+K_{3}(x, y)+\ldots$ with the plane $y=\mu x+a z$ is given by the following formula up to terms of order three:

$$
y(x)=\mu x+a B_{2}(1, \mu) x^{2}+\left[a^{2} \frac{\partial B_{2}}{\partial y}(1, \mu) B_{2}(1, \mu)+a K_{3}(1, \mu)\right] x^{3}+\ldots
$$

The corollary is obtained by applying Lemma 3 to the equation $y=\mu x+a f(x, y)$.

A family of curves on a surface is said to be (locally) rectifiable if there is a (local) diffeomorphism of the surface onto a plane domain that takes each curve in the family to a segment of a straight line. By a pencil of curves centered at a point $A$ we mean an arbitrary family of curves on the surface each of which passes through $A$. If a pencil of curves is locally rectifiable near the center, then different curves of this pencil have different tangents at the center. Let us consider a pencil of curves on the plane, centered at the origin, and denote by $\gamma_{\mu}$ the curve of the pencil that is tangent to the line $y=\mu x$ at the origin. In a neighborhood of the origin, the curve $\gamma_{\mu}$ is a graph of some function $y_{\mu}(x)$.

Lemma 4. If a pencil of curves $\left\{y=y_{\mu}(x)\right\}$ is locally rectifiable near zero, then there exist polynomials $T_{3}$ and $T_{5}$ in the parameter $\mu$ of degrees $\leqslant 3$ and 5 , respectively, such that the Taylor series expansion of the function $y_{\mu}(x)$ up to terms of order three is given by the formula

$$
y_{\mu}(x)=\mu x+T_{3}(\mu) x^{2}+T_{5}(\mu) x^{3}+\ldots
$$

Moreover, the coefficient $b_{5}$ of $\mu^{5}$ in the polynomial $T_{5}$ is related to the coefficient $a_{3}$ of $\mu^{3}$ in the polynomial $T_{3}$ by the formula $b_{5}=2 a_{3}^{2}$.

Proof. Making an affine transformation of the image if necessary, we can assume that the rectifying diffeomorphism takes the origin into itself and has the identity differential at this point, i.e., that it is defined by a pair of functions $G_{1}(x, y)=x+\ldots, G_{2}(x, y)=y+\ldots$, where the dots stand for second-order terms. This diffeomorphism takes the curve $\gamma_{\mu}$ to the straight line $y=\mu x$, i.e., the function $G_{2}(x, y)-\mu G_{1}(x, y)$ vanishes on the curve $\gamma_{\mu}$. Lemma 4 now follows by applying Lemma 3 to this function.

Theorem on seven sections. Assume that the second fundamental form of the germ of the surface at a quadric point is nondegenerate. Let us consider some pencil of sections of the surface by planes passing through the quadric point and transversal to the surface at this point. Assume that this pencil contains at least seven sections. In this case, it is locally rectifiable in a neighborhood of the quadric point if and only if all transversal planes pass through a common straight line transversal to the surface at the quadric point.

Proof. If the pencil of sections is locally rectifiable, then all transversal planes $y=\mu x+a z$ intersect the tangent plane $z=0$ along distinct lines. Therefore, the coefficient $a$ in the equation of the section is a function of the coefficient $\mu, a=g(\mu)$. The function $g$ is defined for the values of the parameter $\mu$ corresponding to the sections in the pencil. If the pencil of sections is locally rectifiable, then, for all these values of the parameter $\mu$, it follows from Corollary 1 and Lemma 4 that the following relations hold: 
(1) $g(\mu) B_{2}(1, \mu)=T_{3}(\mu)$,

(2) $g^{2}(\mu)\left(\partial B_{2} / \partial y(1, \mu)\right) B_{2}(1, \mu)+g(\mu) K_{3}(1, \mu)=T_{5}(\mu)$.

Multiplying relation (2) by $B_{2}$ and using relation (1), we obtain

$$
T_{3}^{2}(1, \mu) \frac{\partial B_{2}}{\partial y}(1, \mu)+T_{3}(\mu) K_{3}(1, \mu)=T_{5}(\mu) B_{2}(1, \mu) .
$$

If the number of sections is not less than seven, then the polynomials on the left- and right-hand sides of relation $(*)$ must be equal identically, since two polynomials of degrees $\leq 7$ with the same leading coefficients identically coincide if they coincide at seven distinct points. Since the center of the pencil is a quadric point, it follows that $K_{3}(1, \mu)$ is divisible by $B_{2}(1, \mu)$. Since the second fundamental form is nondegenerate at the center of the pencil, it follows that the roots of $B_{2}$ are simple, and hence $\left(\partial B_{2} / \partial y\right)(1, \mu)$ and $B_{2}(1, \mu)$ have no common roots. Therefore, it follows from identity $(*)$ that $T_{3}^{2}$ is divisible by $B_{2}$. Since the roots of $B_{2}$ are simple, it follows that $T_{3}$ is divisible by $B_{2}$. It follows from relation $(1)$ that $g(\mu)$ is a linear polynomial in $\mu$. Therefore, all curves of the pencil are cut out on the surface by planes of the form $y=\mu x+(p \mu+q) z$, where $p$ and $q$ are some constants. All these transversal planes pass through a common line that is transversal to the surface at the quadric point.

Conversely, assume that all sections pass through a common line transversal to the surface. In this case, the parallel projection of the surface along this line rectifies the set of sections.

Theorem. Assume that a Riemannian metric on a germ of a nondegenerate quadric in a real projective space is given and that all geodesics with respect to this metric are plane curves. Then all planes containing the geodesics pass through a common point, and the family of all geodesics is locally rectifiable.

Proof. The pencil of geodesics issuing from a point of a Riemannian manifold is locally rectifiable, since it is rectified by the exponential mapping. By the theorem on seven sections, the planes containing the geodesics and passing through a point of the quadric pass through a common line. (This theorem can be applied to geodesics that are not tangent to asymptotic directions, since such a curve cannot be cut out by a tangent plane. For the geodesics tangent to asymptotic directions, we must also use the continuous dependence of a geodesic on the direction.) For sufficiently close points of the quadric, such lines have a nonempty intersection, since sufficiently close points can be joined by a geodesic. Let us choose three points on the quadric that do not belong to the same geodesic. The three lines corresponding to these points intersect each other, and hence pass through a common point, since, by assumption, these three lines do not belong to a common plane. Any line corresponding to a sufficiently close point $A$ on the quadric also passes through this common point, since we can take two of the three chosen points in such a way that there is no geodesic containing these two points together with the point $A$. On a smooth surface belonging to three-dimensional space, the planes passing through a chosen point $O$ of the space cut out a two-parameter family of curves. This family is locally rectifiable about any point $A$ distinct from $O$ for which the line joining $A$ and $O$ is transversal to the surface. Indeed, this family is rectified by the projection from $O$.

Thus, for a given germ of a quadric, the family of plane geodesics is determined by the point $O$ and hence depends on three parameters. Up to a projective transformation of three-dimensional space, there are five such families: three for an elliptic quadric (the point $O$ can be placed either inside the convex body bounded by the quadric, or on its boundary, or outside the body) and two for a hyperbolic quadric (the point $O$ can be placed either on the quadric or outside).

3. Metrics. According to the classical Beltrami theorem (see $[6$, p. 296]), if the geodesics on a plane domain with respect to a Riemannian metric are straight lines, then the metric is of constant curvature. Such a metric is induced, under some projective transformation, by one of the following metrics: the metric of the Klein model of the Lobachevskian geometry, the Euclidean metric of the plane, or the Riemannian metric on the projective plane. (Here we mean the classical metrics, defined up to a positive homothety ratio, whose Gaussian curvature can take any real value.) We thus obtain a complete description of Riemannian metrics on a germ of a nondegenerate quadric for which all geodesics are plane curves. For a given rectifiable family of sections, the Riemannian metric with respect to which these sections are geodesics depends on six parameters, namely, the Gaussian curvature, which can take an arbitrary real value, and an element of the 
eight-dimensional group of the projective transformations of the plane defined modulo the right coset of the three-dimensional subgroup of isometries of the Lobachevskian plane, the Euclidean plane, or the Riemannian plane, respectively.

Corollary 2. (1) A pencil containing at least seven circles or lines on a plane is locally rectifiable if and only if all its curves pass through a common point distinct from the center of the pencil (see [5]).

(2) A two-parameter family of lines and circles on a plane domain is a family of geodesics with respect to some Riemannian metric if and only if this family is induced, under some conformal transformation of the plane (completed by the point at infinity), by the family of geodesics of the Poincaré model of the Lobachevskian plane, by the family of geodesics of the Euclidean plane, or by the family of geodesics of the Riemannian plane. Moreover, a Riemannian metric on the plane domain is induced by the classical metric defined up to a projective transformation.

Proof. Using the stereographic projection, we map the plane onto the sphere. Under this projection, the lines and the circles are mapped into the circles of the sphere, which are plane sections of the sphere. The corollary now follows from the facts proved above.

\section{References}

1. G. S. Khovanskii, Foundations of Nomography [in Russian], Nauka, Moscow, 1976; French transl. G. Khovanski, Éléments de nomographie, éditions Mir, Moscou, 1979.

2. A. G. Khovanskii and G. S. Khovanskii, Dokl. Akad. Nauk SSSR, 248, No. 3, 535-538 (1979).

3. A. G. Khovanskii and G. S. Khovanskii, In: Nomographical Collection [in Russian] (ed. G. S. Khovanskii), No. 14, Academy of Sciences of USSR, Moscow, 1982, pp. 56-77.

4. A. G. Khovanskii and G. S. Khovanskii, In: Nomographical Collection [in Russian] (ed. G. S. Khovanskii), No. 13, Academy of Sciences of USSR, Moscow, 1979, pp. 70-105.

5. A. G. Khovanskii, Sib. Mat. Zh., 21, No. 4, 221-226 (1980).

6. M. P. do Carmo, Differential Geometry of Curves and Surfaces, Prentice-Hall, Englewood Cliffs, NJ, 1976.

Translated by A. I. Shtern 